\title{
A note on common zeroes of Laplace-Beltrami eigenfunctions
}

\author{
V.M. Gichev
}

\begin{abstract}
Let $\Delta u+\lambda u=\Delta v+\lambda v=0$, where $\Delta$ is the Laplace-Beltrami operator on a compact connected smooth manifold $M$ and $\lambda>0$. If $H^{1}(M)=0$ then there exists $p \in M$ such that $u(p)=v(p)=0$. For homogeneous $M, H^{1}(M) \neq 0$ implies the existence of a pair $u, v$ as above that has no common zero.
\end{abstract}

\section{Introduction}

Let $M$ be a compact connected closed orientable $C^{\infty}$-smooth Riemannian $d$-dimensional manifold and $\Delta$ be the Laplace-Beltrami operator on it. Set

$$
E_{\lambda}=\left\{u \in C^{2}(M): \Delta u+\lambda u=0\right\} .
$$

The eigenspace $E_{\lambda}$ can be nontrivial only for $\lambda \geq 0$. If the contrary is not stated explicitly, we assume that functions are real valued and linear spaces are finite dimensional; $H^{p}(M)$ denotes de Rham cohomologies.

Theorem 1. Let $M$ be as above.

(1) Suppose $H^{1}(M)=0$. Then for any $\lambda \neq 0$ and each pair $u, v \in E_{\lambda}$ there exists $p \in M$ such that $u(p)=v(p)=0$.

(2) If $M$ is a homogeneous space of a compact Lie group of isometries then the converse is true: $H^{1}(M) \neq 0$ implies the existence of $\lambda \neq 0$ and $a$ pair $u, v \in E_{\lambda}$ without common zeroes.

The circle $\mathbb{T}=\mathbb{R} / 2 \pi \mathbb{Z}$ and functions $u(t)=\cos t, v(t)=\sin t$ provide the simplest example for (2). Moreover, (2) is an easy consequence of this example and the following observation: for homogeneous Riemannian manifolds $M=G / H$, where $G$ is compact and connected, $H^{1}(M) \neq 0$ is equivalent to 
the existence of $G$-equivariant mapping $M \rightarrow \mathbb{T}$ for some nontrivial action of $G$ on $\mathbb{T}$.

The corollary below gives the answer to the question in [3]: is it true that each orbit of a compact connected irreducible linear group, acting in a complex vector space, meets any hyperplane? I am grateful to P. de la Harpe for making me aware of this question which in fact was the starting point for this note.

Corollary 1. Let $V$ be a complex linear space, $\operatorname{dim} V>1$, and $G \subset \mathrm{GL}(V)$ be a compact connected irreducible group. Then for any $v \in V$ and every linear subspace $H \subset V$ of complex codimension 1 there exists $g \in G$ such that $g v \in H$.

There is a real version of this corollary. Let $\tau$ be a real linear irreducible representation of a compact connected Lie group $G$ in a real linear space $V_{\tau}$. We may assume that $V_{\tau}$ is endowed with the invariant inner product $\langle$,$\rangle and that G$ is equipped with a bi-invariant Riemannian metric. Let $M_{\tau}$ be the space of its matrix elements; by definition, $M_{\tau}$ is the linear span of functions $t_{x y}(g)=\langle\tau(g) x, y\rangle, x, y \in V_{\tau}$. Then either $\tau$ admits an invariant complex structure or its complexification is irreducible. It follows from the Schur lemma that $\Delta u=\lambda_{\tau} u$ for each $u \in M_{\tau}$, where $\Delta$ is the LaplaceBeltrami operator for a bi-invariant metric on $G$. Let us fix $\Delta$ and denote by $\Lambda_{\sigma}$, where $\sigma$ is a finite dimensional real representation, the spectrum of $\Delta$ on $M_{\sigma}$; it is the union of $\lambda_{\tau}$ over all irreducible components $\tau$ of $\sigma$.

Corollary 2. Let $G$ be a compact connected semisimple Lie group, $\sigma$ be as above. Suppose that $\Lambda_{\sigma}$ is a single point $\lambda \neq 0$. Then the orbit of any vector in $V_{\sigma}$ meets each linear subspace of codimension $2 .^{1}$

If $u$ is an eigenfunction of $\Delta$ on a Riemannian manifold $M$ then

$$
N_{u}=\{x \in M: u(x)=0\}
$$

is said to be the nodal set, and connected components of its complement $M_{u}=M \backslash N_{u}$ are called nodal domains. In the following lemma, we formulate the main step in the proof of the theorem (the fact seems to be known but I failed to find a reference).

\footnotetext{
${ }^{1}$ If $\Lambda_{\sigma}$ is not a single point then the assertion is not true. The simplest example is the representation of $\mathrm{SO}(3)$ in the space of harmonic polynomials on $\mathbb{R}^{3}$ of the type $l(x)+q(x)$, where $l$ is linear and $q$ is quadratic. Let us fix $x_{0} \neq 0$ and define a subspace $W$ of codimension 2 by equalities $l\left(x_{0}\right)=0$ and $q\left(x_{0}\right)=0$. If $q_{0}$ is nondegenerate then there exists $l_{0}$ such that $l_{0}^{-1}(0) \cap q_{0}^{-1}(0)=\{0\}$; the orbit of $l_{0}+q_{0}$ does not intersect $W$.
} 
Lemma 1. Let $u, v \in E_{\lambda}, u, v \neq 0$, and let $U, V$ be nodal domains for $u$, $v$, respectively. If $U \subseteq V$ then $u=c v$ for some $c \in \mathbb{R}$.

There are many natural questions concerning the distribution of common zeroes; they seem to be difficult. We prove a very particular result for $d=\operatorname{dim} M=2$. Note that $M$ is diffeomorphic to the sphere $S^{2}$ if $d=2$ and $H^{1}(M)=0$.

Proposition 1. Let $d=2, H^{1}(M)=0, \lambda \neq 0, u \in E_{\lambda}$. Suppose that zero is not a critical value for $u$. Then for each $v \in E_{\lambda}$ every connected component of $N_{u}$ contains at least two points of $N_{v}$.

In fact, each component is a Jordan contour and supports a positive measure which annihilates $E_{\lambda}$.

Let $M$ be the unit sphere $S^{2} \subset \mathbb{R}^{3}$ with the standard metric. Then $\lambda_{n}=$ $n(n+1)$ is $n$-th eigenvalue of $\Delta$. The corresponding eigenspace $E_{n}=E_{\lambda_{n}}$ consists of spherical harmonics which can be defined as restrictions to $S^{2}$ of harmonic (with respect to the ordinary Laplacian in $\mathbb{R}^{3}$ ) homogeneous polynomials of degree $n$ in $\mathbb{R}^{3} ; \operatorname{dim} E_{n}=2 n+1$. The space $E_{n}$ is spanned by zonal spherical harmonics $l_{a, n}(x)=\left.L_{n}(\langle x, a\rangle)\right|_{S^{2}}$, where $a \in S^{2}$ and $L_{n}$ is the Legendre polynomial. The nodal set for $l_{a, n}$ is the union of $n$ circles

$$
\left\{x \in S^{2}:\langle x, a\rangle=x_{k}\right\},
$$

where $x_{1}, \ldots, x_{n} \in[-1,1]$ are zeroes of $L_{n}$. Set $u=l_{a, n}, v=l_{b, n}, n(a, b)=$ $\operatorname{card}\left(N_{u} \cap N_{v}\right)$. Projections of $N_{u}$ and $N_{v}$ to the plane $\pi_{a b}$ passing through $a$ and $b$ are families of segments in the unit disc in $\pi_{a b}$ with endpoints in the unit circle which are orthogonal to $a$ and $b$, respectively. Outside the boundary circle, the preimage of each point is a pair of points. Further, $N_{u}$, $N_{v}$ are symmetric with respect to $\pi_{a b}$. Hence $N_{u} \cap N_{v}$ corresponds to the intersection of the segments. It makes possible to calculate or estimate $n(a, b)$. In particular, if $a$ and $b$ are sufficiently close then $n(a, b)=2 n$; if $a \perp b$ then $n(a, b) \approx c n^{2}$, where $c$ can be calculated explicitly since zeroes of $L_{n}$ are distributed uniformly in $[-1,1]$. The set $N_{u} \cap N_{v}$ can be infinite for independent $u, v \in E_{n}$, for instance, it can be a big circle or a family of parallel circles in $S^{2}$ (this is true for suitable $u, v$ of the type $P(\cos \theta) \cos (k \varphi+\alpha)$, where $P$ is a polynomial, $\theta, \varphi$ are Euler coordinates in $\left.S^{2}, k=1, \ldots, n\right)$. I do not know if there are other nontrivial examples of infinite sets $N_{u} \cap N_{v}$ as well as examples of $u, v \in E_{n}$ such that $\operatorname{card}\left(N_{u} \cap N_{v}\right)<2 n^{2}$.

\footnotetext{
${ }^{2}$ with multiplicities, or for generic $u, v$. If $n=2$ then $4 \leq \operatorname{card}\left(N_{u} \cap N_{v}\right) \leq 8$; for $n=1$, $\operatorname{card}\left(N_{u} \cap N_{v}\right)=2$.
} 
It is natural to ask if something like Theorem 1 is true for three or more eigenfunctions. Here is an example. Let $S^{3}$ be the unit sphere in $\mathbb{C}^{2}$ and set $u=\left|z_{1}\right|^{2}-\left|z_{2}\right|^{2}, v=\operatorname{Re} z_{1} \bar{z}_{2}, w=\operatorname{Im} z_{1} \bar{z}_{2}$. These three Laplace-Beltrami eigenfunctions have no common zeroes in $S^{3}$. They are matrix elements of the three dimensional representation of $\mathrm{SU}(2) \cong S^{3}$ and correspond to three linear functions on $S^{2} \subset \mathbb{R}^{3}$; the homogeneous space $M$ admits an equivariant mapping $M \rightarrow S^{2}$. Perhaps, the latter property could be the right replacement of the assumption $H^{1}(M) \neq 0$ in a version of Theorem 1 for homogeneous spaces and three eigenfunctions.

\section{Proof of results}

By $\rho$ we denote the Riemannian metric in $M, \omega$ is the volume $n$-form. The metric $\rho$ identifies tangent and cotangent bundles, hence it extends to $T^{*} M$. Let $D$ be a domain in $M, C_{c}^{2}(D)$ be the set of all functions in $C^{2}(D)$ with compact support in $D, W_{0}$ be the closure of $C_{c}^{2}(D)$ in the Sobolev class $W_{2}^{1}(D)$ which consist of functions whose first derivatives (in the sense of the distribution theory) are square integrable functions. There is the natural unique up to equivalence norm making it a Banach space. For all $u, v \in C_{c}^{2}(D)^{3}$

$$
\int_{D} \rho(d u, d v) \omega=-\int_{D} u \Delta v \omega=-\int_{D} v \Delta u \omega .
$$

Hence for every $u, v, w \in C_{c}^{2}(D)$

$$
\int_{D} u \rho(d v, d w) \omega=-\int_{D} v(\rho(d u, d w)+u \Delta w) \omega
$$

For a domain $D \subseteq M$ and a function $u \in W_{2}^{1}(D)$, let

$$
\mathcal{D}_{D}(u)=\int_{D} \rho(d u, d u) \omega
$$

be the Dirichlet form. In most cases, we shall omit the index. For the sake of completeness, we give a proof of the classical result which states that a positive eigenfunction corresponds to the first eigenvalue which is multiplicity free. The proof follows [2, Ch. VI, §7].

\footnotetext{
${ }^{3}$ thus, $\Delta=-(d \delta+\delta d)$, where $\delta$ is the adjoint operator for $d$; due to the choice of the sign, $\Delta$ is the ordinary Laplacian in the Euclidean case.
} 
Lemma 2. Let $D$ be a domain in $M, v \in C^{2}(D) \cap W_{0}$. Suppose $v>0$ and $\Delta v+\lambda v=0$ on $D$. Then for all $u \in W_{0}$

$$
\mathcal{D}(u) \geq \lambda \int_{D} u^{2} \omega
$$

and the equality holds if and only if $u=c v$ in $D$ for some $c \in \mathbb{R}$.

Proof. Since $v>0$, each $u \in C_{c}^{2}(D)$ admits the unique factorization $u=\eta v$, where $\eta \in C_{c}^{2}(D)$. Due to (1) and the equality $2 \eta v \rho(d \eta, d v)=v \rho\left(d \eta^{2}, d v\right)$,

$$
\begin{aligned}
\mathcal{D}(u)= & \int_{D} \rho(d(\eta v), d(\eta v)) \omega= \\
& \int_{D}\left(v^{2} \rho(d \eta, d \eta)+2 \eta v \rho(d \eta, d v)+\eta^{2} \rho(d v, d v)\right) \omega= \\
& \int_{D}\left(v^{2} \rho(d \eta, d \eta)+\eta^{2} \rho(d v, d v)\right) \omega-\int_{D} \eta^{2}(\rho(d v, d v)+v \Delta v) \omega= \\
& \int_{D}\left(v^{2} \rho(d \eta, d \eta)+\lambda \eta^{2} v^{2}\right) \omega \geq \lambda \int_{D} \eta^{2} v^{2} \omega=\lambda \int_{D} u^{2} \omega .
\end{aligned}
$$

Using the approximation, we get (2). Suppose that the equality in (2) holds for some $u \in W_{0}$. Let $\eta_{n}$ be such that $\eta_{n} v \rightarrow u$ in $W_{0}$ as $n \rightarrow \infty$. Then $\mathcal{D}\left(\eta_{n} v\right) \rightarrow \mathcal{D}(u)$. Due to the calculation above,

$$
\lim _{n \rightarrow \infty} \int_{D} v^{2} \rho\left(d \eta_{n}, d \eta_{n}\right) \omega=0
$$

Let $D^{\prime} \subset D$ be a domain whose closure is contained in $D$. Standard arguments show that any limit point of the sequence $\left\{\eta_{n}\right\}$ in $W_{2}^{1}\left(D^{\prime}\right)$ is a constant function. Hence $u=c v$ in $D$ for some $c \in \mathbb{R}$. The converse is obvious.

Proof of Lemma 1. Let $D=V \supseteq U$; we may assume $v>0$ in $D$ and $u>0$ in $U$. Let $\tilde{u}$ be zero outside $U$ and coincide with $u$ in $U$. Clearly, ${ }^{4} v, \tilde{u} \in W_{0}$. Furthermore,

$$
\mathcal{D}_{D}(\tilde{u})=\mathcal{D}_{U}(u)=\lambda \int_{U} u^{2} \omega=\lambda \int_{D} \tilde{u}^{2} \omega .
$$

By Lemma 2, $u=c v$ in $D$. To conclude the proof, we refer to Aronszajn's unique continuation theorem [1] which implies $u=c v$ on $M$.

\footnotetext{
${ }^{4} \tilde{u}$ can be approximated in $W_{0}$ by functions $u_{n}=\max \left\{\varepsilon_{n}, u\right\}-\varepsilon_{n}$ in $U, u_{n}=0$ outside $U$, where $\varepsilon_{n}>0$ are regular values for $u$ and $\varepsilon_{n} \rightarrow 0$ as $n \rightarrow \infty$ (note that $u \in C^{1}(M)$ ).
} 
Proof of Theorem 1. 1) Let $\mathfrak{U}$ and $\mathfrak{V}$ be families of nodal domains for $u$ and $v$, respectively. The assumption $\lambda \neq 0$ and the orthogonality relations imply $M_{u}, M_{v} \neq M$. Obviously, $u$ and $v$ have no common zeroes if and only if $\mathfrak{C}=\mathfrak{U} \cup \mathfrak{V}$ is a covering:

$$
M=\bigcup_{W \in \mathfrak{C}} W
$$

It is sufficient to prove, assuming (3), that there exists a closed 1-form on $M$ which is not exact. The covering $\mathfrak{C}$ has following properties:

(A) sets in $\mathfrak{U}$ are pairwise disjoint, and the same is true for $\mathfrak{V}$;

(B) nor $U \subseteq V$ neither $U \supseteq V$ for every $U \in \mathfrak{U}, V \in \mathfrak{V}$.

The first is obvious, the second is a consequence of Lemma 1. Also, Lemma 1 implies that

$$
U \cap N_{v} \neq \emptyset, \quad V \cap N_{u} \neq \emptyset \quad \text { for all } U \in \mathfrak{U}, V \in \mathfrak{V} .
$$

Due to (4), $\mathfrak{C}$ is finite: $\mathfrak{V}$ covers the compact set $N_{u}$ by open disjoint sets, and the same is true for $\mathfrak{U}$ and $N_{v}$. This also means that a connected component $X$ of $N_{u}$ is contained in some nodal domain for $v$. Further, $u$ cannot keep its sign near $X$. Otherwise, we get a contradiction assuming $u>0$ and applying the Green formula to functions $u, 1$ and the component of the set $u<\varepsilon$ which contains $X$ (for sufficiently small regular $\varepsilon>0$ ) ${ }^{5}$ Hence $X$ lies in the boundary of at least two domains in $\mathfrak{U}$. Components of $N_{v}$ have this property with respect to $\mathfrak{V}$. Let $\Gamma$ be the incidence graph for $\mathfrak{C}$ whose family of vertices is $\mathfrak{C}$ and edges join sets with nonempty intersection. For $\Gamma$, the conditions above read as follows:

(a) each edge of $\Gamma$ joins $\mathfrak{U}$ and $\mathfrak{V}$;

(b) any vertex is common for (at least) two different edges ${ }^{6}$.

\footnotetext{
${ }^{5}$ Indeed, $\int_{\partial U_{\varepsilon}} \delta(u \omega)=\int_{U_{\varepsilon}} d(\delta(u \omega))=-\int_{U_{\varepsilon}} \Delta u \omega=\lambda \int_{U_{\varepsilon}} u \omega>0$, where the operator $\delta$ is adjoint to $d$ and $U_{\varepsilon} \supset X$ is the component above. On the other hand, $\delta(u \omega)=-\frac{\partial u}{\partial n} \omega_{\varepsilon}$, where $\frac{\partial}{\partial n}$ is the outer normal and $\omega_{\varepsilon}$ is the volume form for $\partial U_{\varepsilon}$. Since $\frac{\partial u}{\partial n} \geq 0$ on $\partial U_{\varepsilon}$, we get a contradiction.

${ }^{6}$ Otherwise, there exist $U \in \mathfrak{U}, V \in \mathfrak{V}$ such that $U \subseteq \operatorname{clos} V$ or $V \subseteq \operatorname{clos} U$. If $U \subseteq \operatorname{clos} V=V \cup \partial V$ then either $U \subseteq V$ or $U \cap \partial V \neq \emptyset$. The first contradicts to (B), the second implies the existence of a component $X$ of $N_{v}$ such that $v$ keeps its sign near $X$.
} 
It follows that $\Gamma$ contains a nontrivial cycle $C$. Let $U \in \mathfrak{U}$ and $V \in \mathfrak{V}$ be consecutive vertices of $C, Q=\partial U \cap V$. Since $Q \cap \partial V=\emptyset$ due to (3), both $Q$ and $\partial U \backslash Q=\partial U \backslash V$ are compact. Hence there exists a smooth function $f$ on $M$ such that $f=1$ in a neighbourhood of $Q$ and $f=0$ near $\partial U \backslash Q$. Then $d f=0$ on $\partial U$ and the 1-form $\eta$ which is zero outside $U$ and coincides with $d f$ on $U$ is well defined and smooth. Obviously, $\eta$ is closed; we claim that $\eta$ cannot be exact.

Suppose $\eta=d F$. Then $F=$ const on each connected set which does not intersect $\operatorname{supp} \eta \subset U$. Let $U_{1}=U, V_{1}=V, \ldots, U_{m}, V_{m}$ be the cycle $C$. Then $m>1$ and we may assume that

$$
V_{k} \cap U=\emptyset \text { for } 1<k<m
$$

replacing $C$ by a shorter cycle if necessary ${ }^{7}$. If a curve in $V$ starts outside $U$ and comes into $U$ then it meets $U$ at a point of $Q$. Hence there exists a curve $c_{1}$ in $V \backslash U$ with endpoints in $Q$ and $U_{2}$. Analogously, there is a curve $c_{2}$ inside $V_{m} \backslash U$ which joins a point in $U_{m}$ with a point in $V_{m} \cap \partial U$. The set

$$
X=c_{1} \cup U_{2} \cup V_{2} \cup \ldots \cup U_{m} \cup c_{2}
$$

is connected; by A) and (5), $X \cap U=\emptyset$. Therefore, $F$ is constant on $X$. This contradicts to the choice of $f$ since the closure of $X$ has common points with $Q$ and $\partial U \backslash Q$ (recall that $d F=d f$ on $U$ and that $f$ takes different values on these sets).

2) Let $M=G / H$, where $G$ is a compact group of isometries. Since $M$ is connected, the identity component of $G$ acts on $M$ transitively. Hence we may assume that $G$ is connected. If $H^{1}(M) \neq 0$ then there exists an invariant closed 1-form $\eta$ on $M$ that is not exact. It can be lifted to the left invariant closed 1 -form $\tilde{\eta}$ on the universal covering group $\tilde{G}$. Since $\tilde{\eta}$ is left invariant and closed (hence exact), $\tilde{\eta}=d \chi$ for some nontrivial additive character $\chi: \tilde{G} \rightarrow \mathbb{R}$. According to structure theorems, $\tilde{G}=\tilde{S} \times \mathbb{R}^{k}$, where $\tilde{S}$ is compact, simply connected, and semisimple. Hence $\chi=0$ on $\tilde{S}$. Further, $\eta$ is locally exact on $M$; thus $\chi=0$ on the preimage $\tilde{H}$ of the group $H$ in $\tilde{G}$. Since $\tilde{S}$ is compact and normal, $\tilde{L}=\tilde{S} \tilde{H}$ is a closed subgroup of $\tilde{G}$. It follows that $\operatorname{dim} \tilde{L}<\operatorname{dim} G$. Let $S, L$ be subgroups of $G$ which are covered by $\tilde{S}, \tilde{L}$, respectively. Thus, $L=S H$ is a closed proper subgroup of $G$. The natural mapping $M=G / H \rightarrow G / L \cong \mathbb{T}^{m}$ can be continued to a nontrivial equivariant one $M \rightarrow \mathbb{T}$. Realizing $\mathbb{T}$ as the unit circle in $\mathbb{C}$ we get a nonconstant function whose real and imaginary parts satisfies the theorem.

\footnotetext{
${ }^{7}$ We may assume that $C$ contains no proper subcycle.
} 
Conditions A) and B) imply $H^{1}(\mathfrak{C}, A) \neq 0$ for any nontrivial abelian group $A$. Indeed, every three distinct sets in $\mathfrak{C}$ have empty intersection whence any function on the set of edges of $\Gamma$ is a cocycle, while for each coboundary the sum of its values along every cycle in $\Gamma$ is zero. Besides, for homogeneous spaces of connected compact Lie groups the condition $H^{1}(M)=0$ is equivalent to each of following ones: $H_{1}(M, \mathbb{Z})$ is finite; $\pi_{1}(M)$ is finite; $\tilde{M}$ is compact; the semisimple part of $G$ acts on $M$ transitively; $\mathfrak{g}=\mathfrak{h}+[\mathfrak{g}, \mathfrak{g}]$, where $\mathfrak{g}$ and $\mathfrak{h}$ are Lie algebras of $G$ and $H$, respectively. We omit the proof which is easy.

Proof of Corollary 2. Since $G$ is semisimple, $H^{1}(G)=0$. Let $L \subset V_{\sigma}$ be a subspace of codimension $2, x \in V_{\sigma}, y$ and $z$ be a linear base of $L^{\perp}$; set $u(g)=\langle\sigma(g) x, y\rangle, v(g)=\langle\sigma(g) x, z\rangle$. By Theorem $1, u$ and $v$ have a common zero $g \in G$; then $\sigma(g) x \perp y, z$ but this is equivalent to $\sigma(g) x \in L$.

Proof of Corollary 1. Clearly, the semisimple part of $G$ is irreducible. Hence $G$ can be assumed to be semisimple. The condition $\operatorname{dim} V>1$ implies that the representation is not trivial. Therefore, $\Lambda_{\tau}$ is a single point $\lambda_{\tau} \neq 0$ and the hyperplane $H$ has the real codimension 2 in $V$. Thus we may apply Corollary 2.

Note that the centre of $G$ consists of scalar matrices; hence, if $G$ is not semisimple then $H \cap G v$ includes $\mathbb{T} v$ for any $v \in H$, where $\mathbb{T}$ is the unit circle in $\mathbb{C}$. Therefore, $H \cap G v$ is infinite for any $v \in V \backslash\{0\}$ in this case.

In what follows, we assume that $M$ is diffeomorphic to the sphere $S^{2}$. Let $D$ be a domain in $M$ bounded by a finite number of smooth curves. Then there exists a vector field $\frac{\partial}{\partial n}$ on $\partial D$ orthogonal to $\partial D$ such that the Green formula holds:

$$
\int_{\partial D}\left(u \frac{\partial v}{\partial n}-v \frac{\partial u}{\partial n}\right) d s=\int_{D}(u \Delta v-v \Delta u) d m
$$

for all smooth $u, v$, where $s$ and $m$ are linear and area measures defined by $\rho$ on $\partial D$ and $D$, respectively. The vector field $\frac{\partial}{\partial n}$ depends only on the local geometry of $\partial D$ and does not vanish on $\partial D$.

Lemma 3. Let $\lambda \neq 0, u \in E_{\lambda}$, and $C$ be a component of $N_{u}$. If $C$ is a Jordan contour that contains no critical points of $u$ then there exists a strictly positive continuous function $q$ on $C$ such that $\int_{C} v q d s=0$ for all $v \in E_{\lambda}$. 
Proof. Since $d u \neq 0$ on $C$, it is a smooth curve. Let $D$ be one of the two domains bounded by $C$ due to Jordan Theorem. Applying (6) to it, we get

$$
\int_{C} v \frac{\partial u}{\partial n} d s=0
$$

Clearly, if $\frac{\partial u}{\partial n}(p)=0$ for $p \in C$ then $p$ is a critical point. Hence either $q=\frac{\partial u}{\partial n}$ or $q=-\frac{\partial u}{\partial n}$ satisfies the lemma.

Proposition 1 is an easy consequence of Lemma 3 (it remains to note that each component of $N_{u}$ is a Jordan contour if 0 is not a critical value).

\section{References}

[1] Aronszajn N., A unique continuation theorem for solutions of elliptic partial differential equations of second order, J. Math. Pures Appl., 36 (1957), 235-239.

[2] Courant R., Hilbert D., Methoden der Mathematischen Physik, Berlin, Verlag von Julius Springer, 1931.

[3] Galindo J., de la Harpe P., Vust T., Two Observations on Irreducible Representations of Groups, J. of Lie Theory, 12 (2002), 535-538.

V.M. Gichev

gichev@iitam.omsk.net.ru

Omsk Branch of

Sobolev Institute of Mathematics

Pevtsova, 13, 644099

Omsk, Russia 